\title{
Low pericardial fluid glucose concentration combined with computed tomography evaluation as a malignancy-related marker in symptomatic patients undergoing pericardiocentesis: a retrospective study
}

Takashi Nakamura

Kindai University

Mana Okune

Kindai University

Masakazu Yasuda

Kindai University

Heitaro Watanabe

Kindai University

Masafumi Ueno

Kindai University

Kenji Yamaji

Kindai University

Kazuki Mizutani

Kindai University

Takashi Kurita

Kindai University

Gaku Nakazawa ( $\sim$ gnakazawa@med.kindai.ac.jp )

Kindai University

\section{Research Article}

Keywords: pericardial effusion, pericardial fluid glucose level, malignancy, CT attenuation values

Posted Date: March 11th, 2021

DOI: https://doi.org/10.21203/rs.3.rs-275585/v1

License: (c) (i) This work is licensed under a Creative Commons Attribution 4.0 International License.

Read Full License 


\section{Abstract}

Background: We evaluated malignancy according to the characteristics of pericardial fluid in symptomatic Japanese patients undergoing pericardiocentesis and computed tomography (CT).

Methods: This was a retrospective, single-center, observational study of 125 symptomatic patients undergoing pericardiocentesis. The patients were classified into two groups: a malignancy group and a non-malignancy group, according to the primary disease and cytology of the pericardial effusion (PE). We compared the pericardial fluid sample and CT measurements between both groups.

Results: All patients were diagnosed as having exudative PE by Light's criteria. PE with malignant cells was demonstrated in $76.8 \%$ of the malignancy group patients. A ratio of pericardial to serum lactate dehydrogenase $(\mathrm{LDH})>0.6$ as one of Light's criteria, and lower serum brain natriuretic peptide (BNP: $126.9 \pm 89.8 \mathrm{pg} / \mathrm{ml}$ vs $409.2 \pm 97.7 \mathrm{pg} / \mathrm{ml}$, malignancy vs non-malignancy groups, respectively; $p=0.037$ ) were associated with malignancy (both, $p<0.05$ ). A significant difference was observed in pericardial fluid glucose level between the malignancy and non-malignancy groups (pericardial fluid glucose: $78.25 \pm 48.29 \mathrm{mg} / \mathrm{dl}$ vs $98.41 \pm 44.85$, respectively; $p<0.05$ ). Moreover, CT attenuation values (Hounsfield units $(\mathrm{HU}))$ tended to be higher in the malignancy group vs the non-malignancy group (22.7 [interquartile range (IQR), 17.4-26.0] vs 17.4 [IQR, 13.7-26.4], respectively; $p=0.08$ ). Pericardial fluid glucose level $\leq 70 \mathrm{mg} / \mathrm{dl}$ and $\mathrm{CT}$ attenuation values $>20 \mathrm{HU}$ were strongly associated with malignancy.

Conclusions: Lower pericardial fluid glucose level with higher CT attenuation values may suggest malignancy-related PE.

\section{Background}

The normal pericardial sac contains up to $50 \mathrm{ml}$ of pericardial fluid as a plasma ultrafiltrate. Pericardial effusion (PE), which is classified according to composition, distribution, and volume, results from many diseases [1]. In patients with $P E$, the prevalence of malignancy or infection ranges from $15-50 \%[2,3]$, and malignant PE can be diagnosed by cytology. In addition to imaging and pericardial fluid analysis, the clinical course of the underlying disease can contribute to the diagnosis of malignancy-related PE. In particular, large $\mathrm{PE}$ is a common manifestation in malignant disease, and patients with malignant PE have a poor prognosis because of advanced-disease stage [4, 5]. In cases without pre-existing cancer, malignancy must be excluded because PE may be the first manifestation of malignancy. Some studies reported PE attributed to unknown causes at a rate of $10-20 \%[5,6]$. It may be difficult to diagnose malignant PE even if cytology or epicardial and pericardial biopsy are performed [7]. A previous study of pericardial fluid analysis showed that diseases with exudative PE had lower pericardial fluid glucose levels compared with diseases with transudative PE [8]. However, the relationship between malignancyrelated PE and non-malignant PE evaluated using computed tomography (CT) to analyze the pericardial fluid is unknown. Therefore, we examined malignancy according to the characteristics of the pericardial fluid and CT measurements in symptomatic patients undergoing pericardiocentesis. 


\section{Methods}

Patients

We enrolled 125 consecutive symptomatic patients undergoing pericardiocentesis between April 2010 and April 2020 as a retrospective observational study in a single institution.

Twenty-eight patients without pericardial fluid sampling and CT data were excluded because of out-ofhospital cardiopulmonary arrest, Stanford type A aortic dissection, and blow-out rupture in acute myocardial infarction as an intra-procedural complication. Therefore, 97 patients were finally enrolled in this study. The patients were classified into two groups: a malignancy group and a non-malignancy group. The malignancy group constituted patients with known existing malignancy or documented malignant cells in drained PE.

Blood samples and pericardial fluid samples

Blood samples were collected approximately 24 hours after drainage. We evaluated the following in the drained pericardial fluid: $\mathrm{pH}$, total protein, lactate dehydrogenase (LDH), albumin, adenosine deaminase $(\mathrm{ADA})$, hyaluronic acid, hemoglobin $(\mathrm{Hb})$, and hematocrit. Collected pericardial fluid was cytologically diagnosed with malignant cells or non-malignant cells by two pathologists. Pericardial fluid testing was performed in 88 patients, and cytological analysis was performed in 94 patients. Patients were diagnosed with exudative PE according to Light's criteria, which constitute a pericardial fluid-to-serum total protein ratio $>0.5$ and a pericardial fluid-to-serum LDH ratio $>0.6$ or two-thirds of the upper limit of the normal (UNL) serum LDH value.

CT Protocol and measurement

CT scans before drainage were performed within 24 hours of admission using a 64-multidetctor CT scanner (Discovery HD 750; GE Healthcare, Milwaukee, WI, USA). Eighty-one patients were evaluated, with 5 -mm slice thickness, gantry rotation speed of $350 \mathrm{~ms}, 64 \times 0.625-\mathrm{mm}$ collimation, tube voltage of 120 $\mathrm{kV}$, and effective tube current of 325-750 mA. Axial images were transferred to an available workstation and analyzed using SYNAPSE VINCENT software (Fujifilm ${ }^{\text {TM }}$ Co., Tokyo, Japan). The CT attenuation values in the PE were calculated and analyzed by SYNAPSE VINCENT [9]. In addition to a previous method that was determined by the region of interest (ROI), CT attenuation values and pericardial area were measured by plotting around the PE using a two-dimensional (2D) image analysis system in three axial view slices as follows: 1) upper slice: bifurcation of the pulmonary trunk, 2) middle slice: fourchamber heart view, and 3) lower slice: upper edge of the liver (Fig. 1) [10]. The average CT attenuation value from the three slices was calculated as Hounsfield units (HU). The measurements were reanalyzed by the same interpreters to evaluate intraobserver agreement.

\section{Statistical analysis}


Categorical variables were compared using the $\chi^{2}$ test for proportions and the unpaired t-test or analysis of variance for continuous variables, as appropriate. The linearity of the relationship between two variables was assessed by linear regression analysis. Correlation analysis was performed with Pearson's correlation coefficient. Parameters with non-normal distributions were analyzed using the Mann-Whitney $U$ test. $p<0.05$ was considered significant, and results are expressed as the mean \pm standard deviation (SD). All analyses were performed using JMP version 11.0.

\section{Results}

Baseline clinical characteristics

The baseline characteristics of the 97 subjects are shown in Table 1; the majority were male and $>65$ years of age. Approximately $10 \%$ of the patients had previously taken antiplatelet agents, but few patients had received prior anticoagulation therapy. The most frequent etiology in patients undergoing pericardiocentesis was malignant disease (58.8\%), and among these patients, $59.6 \%$ had lung cancer (Table 2). Pericardial fluid with malignant cells: positive-cytology was demonstrated in $76.8 \%$ of the patients in the malignancy group. 
Table 1

The patients' baseline characteristics

\begin{tabular}{|ll|}
\hline Characteristic & $\mathbf{n}=97$ \\
\hline Age (years) & $66.1 \pm 14.2$ \\
\hline Men (\%) & $58(59.8)$ \\
\hline Medications (\%) & $10(10.3)$ \\
\hline Prior antiplatelet & $6(6.2)$ \\
\hline Prior novel oral anticoagulants & $5(5.2)$ \\
\hline Prior warfarin & \\
\hline Symptom (\%) & $84(86.6)$ \\
\hline Dyspnea & $15(15.5)$ \\
\hline Cough & $10(10.3)$ \\
\hline Anorexia & $9(9.3)$ \\
\hline Chest pain & $5(5.2)$ \\
\hline Syncope & $550.87 \pm 262.31$ \\
\hline Primary drained pericardial effusion (ml) & \\
\hline Etiology (\%) & $1(1.0)$ \\
\hline Malignancy & $57(58.8)$ \\
\hline Collagen vascular diseases & $8(8.2)$ \\
\hline Idiopathic & $7(7.2)$ \\
\hline Infection & $5(5.1)$ \\
\hline Post-myocardial infarction & $4(4.1)$ \\
\hline Uremia & $3(4.1)$ \\
\hline latrogenic & $3(3.1)$ \\
\hline Post-surgery & $1(3.1)$ \\
\hline Chronic graft-versus-host disease & \\
\hline Radiation & \\
\hline Dissection & \\
\hline Hypothyroidism & \\
\hline The baseline clinical characteristics of all & \\
\hline
\end{tabular}


Table 2

Etiologies in the malignancy group

\begin{tabular}{|l|l|}
\hline Malignancy & $\mathbf{n = 5 7}$ \\
\hline Lung cancer & $34(59.6)$ \\
\hline Gastrointestinal carcinoma & $7(12.3)$ \\
\hline Malignant lymphoma & $3(5.3)$ \\
\hline Renal carcinoma & $3(5.3)$ \\
\hline Breast carcinoma & $2(3.5)$ \\
\hline Gynecological carcinoma & $2(3.5)$ \\
\hline Pleural mesothelioma & $1(1.8)$ \\
\hline Hemangiosarcoma & $1(1.8)$ \\
\hline Primary cancer of unknown type & 4 (7.0) \\
\hline Among patients with malignant disease, patients with lung cancer constituted the highest percentage. \\
\hline
\end{tabular}

Blood samples (Table 3)

No significant differences in serum C-reactive protein (CRP), total protein, $\mathrm{LDH}$, albumin, glucose, and $\mathrm{Hb}$ were observed between the malignancy and non-malignancy groups. In contrast, estimated glomerular filtration rate (eGFR) was significantly higher in patients with vs without malignancy $(60.56 \pm 31.42$ $\mathrm{ml} / \mathrm{min} / 1.73 \mathrm{~m}^{2}$ vs $45.97 \pm 29.08 \mathrm{ml} / \mathrm{min} / 1.73 \mathrm{~m}^{2}$, respectively; $\left.p=0.024\right)$. Serum brain natriuretic peptide (BNP) levels were significantly lower in patients with vs without malignancy $(126.89 \pm 183.35 \mathrm{pg} / \mathrm{ml}$ vs $409.18 \pm 868.67 \mathrm{pg} / \mathrm{ml}$, respectively; $p=0.037$ ). 
Table 3

Comparison between the malignancy and non-malignancy groups

\begin{tabular}{|llll|}
\hline Serum component & $\begin{array}{l}\text { Malignancy } \\
\text { Group }\end{array}$ & $\begin{array}{l}\text { Non-Malignancy } \\
\text { Group }\end{array}$ & $p$ \\
\hline CRP $(\mathrm{mg} / \mathrm{dl})$ & $5.33 \pm 4.37$ & $4.54 \pm 6.70$ & 0.488 \\
\hline eGFR (ml/min/1.73m²) & $60.56 \pm 31.42$ & $45.97 \pm 29.08$ & 0.024 \\
\hline Total protein (g/dl) & $6.08 \pm 0.60$ & $6.06 \pm 1.27$ & 0.889 \\
\hline LDH (U/L) & $543.35 \pm 767.60$ & $357.98 \pm 186.54$ & 0.138 \\
\hline Albumin (g/dl) & $3.01 \pm 0.46$ & $3.11 \pm 0.75$ & 0.399 \\
\hline Glucose (mg/dl) & $142.20 \pm 61.03$ & $134.10 \pm 53.05$ & 0.518 \\
\hline Hemoglobin (g/dl) & $11.01 \pm 1.77$ & $11.11 \pm 1.60$ & 0.766 \\
\hline Hematocrit (\%) & $33.29 \pm 5.61$ & $34.24 \pm 4.84$ & 0.391 \\
\hline BNP (pg/ml) & $126.89 \pm 183.35$ & $409.18 \pm 868.67$ & 0.037 \\
\hline Pericardial fluid component & & & \\
\hline Ph & $7.39 \pm 0.20$ & $7.48 \pm 0.12$ & 0.069 \\
\hline Total protein (g/dl) & $5.02 \pm 0.80$ & $4.72 \pm 1.19$ & 0.166 \\
\hline LDH (U/L) & $2032.10 \pm$ & $1029.58 \pm 1561.52$ & 0.106 \\
\hline Albumin (g/dl) & 3522.33 & & 0.661 \\
\hline Glucose (mg/dl) & $2.66 \pm 0.53$ & $2.61 \pm 0.70$ & 0.048 \\
\hline ADA (U/L) & $78.24 \pm 48.29$ & $98.41 \pm 44.85$ & 0.389 \\
\hline Hyaluronic acid (ng/ml) & $37.59 \pm 47.26$ & $29.34 \pm 37.66$ & 0.438 \\
\hline Hemoglobin (g/dl) & $31975.9 \pm$ & $27194.6 \pm 28745.9$ & \\
\hline Hematocrit (\%) & 27282.7 & $5.15 \pm 5.19$ & \\
\hline Variable & $3.83 \pm 2.22$ & $16.74 \pm 21.36$ & \\
\hline Pericardial fluid/Serum ratio & $14.79 \pm 6.73$ & & \\
\hline
\end{tabular}

Lower serum BNP and lower pericardial fluid glucose level were associated with malignancy.

Values are expressed as mean \pm standard deviation

$C R P$ C-reactive protein, $e G F R$ estimated glomerular filtration rate, $L D H$ lactate dehydrogenase, $B N P$ brain natriuretic protein, $A D A$ adenosine deaminase, $U N L$ upper limit of normal 


\begin{tabular}{|llll|}
\hline Serum component & $\begin{array}{l}\text { Malignancy } \\
\text { Group }\end{array}$ & $\begin{array}{l}\text { Non-Malignancy } \\
\text { Group }\end{array}$ & $p$ \\
\hline Total protein ratio & $0.83 \pm 0.14$ & $0.78 \pm 0.15$ & 0.127 \\
\hline LDH ratio & $5.23 \pm 7.69$ & $2.59 \pm 2.71$ & 0.047 \\
\hline Glucose ratio & $0.57 \pm 0.34$ & $0.83 \pm 0.45$ & 0.005 \\
\hline $\begin{array}{l}\text { Pericardial fluid/Serum total protein ratio }> \\
0.5\end{array}$ & $49(100 \%)$ & $38(97.4 \%)$ & 0.260 \\
\hline Pericardial fluid/Serum LDH ratio > 0.6 & $47(94.0 \%)$ & $29(76.3 \%)$ & 0.017 \\
\hline Pericardial fluid LDH > 2/3 UNL & $49(98.0 \%)$ & $32(86.5 \%)$ & 0.036 \\
\hline Cytology, positive & $43(76.8 \%)$ & - & - \\
\hline \begin{tabular}{l} 
Lower serum BNP and lower pericardial fluid glucose level were associated with malignancy. \\
\hline Values are expressed as mean \pm standard deviation
\end{tabular} & \\
\hline $\begin{array}{l}\text { CRP C-reactive protein, } e G F R \text { estimated glomerular filtration rate, } L D H \text { lactate dehydrogenase, } B N P \\
\text { brain natriuretic protein, } A D A \text { adenosine deaminase, UNL upper limit of normal }\end{array}$ \\
\hline
\end{tabular}

Pericardial fluid samples, pericardial/serum ratio, Light's criteria, and cytology (Table 3).

No significant differences in pericardial fluid pH levels, total protein, $\mathrm{LDH}$, albumin, ADA, and hyaluronic acid were observed between the two groups. Pericardial fluid glucose levels were significantly lower in patients with vs without malignancy $(78.24 \pm 48.29$ vs $98.41 \pm 44.85$, respectively; $p=0.048)$ (Fig. 2$)$.

Malignant cells in the pericardial fluid was demonstrated in $76.8 \%$ of the patients in the malignancy group. All patients were diagnosed as having exudative PE by Light's criteria. Of the three items in Light's criteria, $L D H$ ratio $>0.6$ and pericardial $L D H>2 / 3$ of the UNL were associated with malignancy $(p=0.017$ and $p=0.036$, respectively). The pericardial fluid-to-serum LDH ratios were significantly higher in patients with malignancy than in those without malignancy ( $5.23 \pm 7.69$ vs $2.59 \pm 2.71$, respectively; $p=0.047$ ). The pericardial fluid-to-serum glucose ratios were significantly lower in patients with vs without malignancy ( $0.57 \pm 0.34$ vs $0.83 \pm 0.45$, respectively; $p=0.005)$ (Fig. 2$)$.

CT measurements

No significant differences in CT parameters were observed between the malignancy and non-malignancy groups. However, CT attenuation values (HU) tended to be higher in the malignancy group compared with the non-malignancy group (22.7 [interquartile range (IQR), 17.4-26.0] vs 17.4 [IQR, 13.7-26.4], respectively; $p=0.08$ ).

The relationship between pericardial fluid samples and CT measurements 
The malignancy group had a higher median CT attenuation value of $20.6 \mathrm{HU}(p<0.05)$. Moreover, overall, CT attenuation values $>20 \mathrm{HU}$ were associated with malignancy $(p<0.05)$ (Fig. 3$)$. The relationship between pericardial fluid measurements and CT attenuation values is shown in Fig. 4. CT attenuation values were strongly correlated with pericardial fluid hematocrit $(r=0.908)$. CT attenuation values were also correlated with pericardial fluid glucose level $(r=-0.425)$, protein level $(r=0.541)$, glucose ratio $(r=-$ $0.461)$, and protein ratio $(r=0.583)$. Pericardial fluid glucose $\leq 70 \mathrm{mg} / \mathrm{dl}$ and CT attenuation values $>20$ $\mathrm{HU}$ were cutoff values associated with malignancy (Fig. 5). Additionally, pericardial fluid glucose $\leq 70$ $\mathrm{mg} / \mathrm{dl}$ and $\mathrm{CT}$ attenuation values $>20 \mathrm{HU}$ were also cutoff values associated with positive-cytology patients (see Supplementary Fig. 1, Additional File 1).

\section{Discussion}

In the current study, we investigated the characteristics of malignant pericardial fluid in symptomatic patients undergoing pericardiocentesis and CT; lower pericardial fluid glucose level and higher CT attenuation values were related to malignancy. Strobbe et al. evaluated the etiologies of PE and distinguished radiation therapy for malignancy as a cause of PE [11]. Moreover, they reported that bone marrow transplantation was an etiology in the idiopathic group [11]. Additionally, Sullivan et al. reported that chronic graft-versus-host disease (GVHD) presented as an immune reaction after bone marrow transplantation, similar to the mechanism in autoimmune diseases [12]. Therefore, non-recurrent cancer patients with prior radiation therapy or chronic GVHD were included in our non-malignancy group. In the current study, the number of patients in the malignancy group was higher than the number in the nonmalignancy group, and approximately $60 \%$ of the patients in the malignancy group had lung cancer.

One study in Western Asia and another in Africa reported PE attributed to unknown causes at rates of approximately $10-15 \%[6,13]$. In contrast, a study in Western Europe and another in North America reported that approximately $80-90 \%$ of patients with PE were classified as having an idiopathic etiology $[14,15]$. Distinguishing malignant PE from non-malignant $P E$ has important implications regarding prognosis. However, two previous studies reported that 44.9-92\% of malignant patients often demonstrated malignant cells in PE $[8,16]$. As a result of cytology by repeat drainage, the current study showed that $76.8 \%$ of patients in the malignancy group demonstrated malignant cells in the pericardial fluid. Standard pericardial cytology does not necessarily exclude malignant PE; therefore it is necessary to detect neoplasia using blood samples, pericardial fluid analysis, and imaging modalities, such as positron-emission tomography-CT (PET-CT). Cytology-negative patients in the malignancy group in this study were defined as patients who had untreatable recurrent or metastatic carcinoma.

Although pericardial fluid analysis is performed routinely, the results of these analysis are not effectively utilized owing to a lack of specificity. Meyers et al. reported that pericardial fluid glucose level was lower in patients with exudates than in those with transudates, and there was a significant overlap in the etiology of PE between patients with transudates vs exudates [8]. All cases in our study, in both the malignancy and non-malignancy groups, were diagnosed as having exudative PE by Light's criteria. No significant differences in pericardial fluid LDH levels were observed between the groups, while LDH ratio > 
0.6 and pericardial LDH $>2 / 3$ of the UNL were associated with malignancy. Ben-Horin et al. demonstrated pericardial fluid LDH level $>2.4$ times the serum level and mean pericardial fluid protein level 0.6 times the serum level in patients undergoing elective open surgery without a history of pericardial diseases [17]. Despite these findings, a recent study showed that malignant patients had lower PE LDH levels than nonmalignant patients, and another study showed that malignant patients had lower PE glucose levels compared with patients with heart failure-related effusion $[16,18]$. Furthermore, Ben-Horin et al. demonstrated a mean pericardial fluid glucose level of $133 \mathrm{mg} / \mathrm{dl}$ and mean pericardial fluid-to-serum glucose ratio of 1.0 in patients without a history of pericardial diseases [17]. In the current study, pericardial fluid glucose levels and pericardial fluid-to-serum glucose ratio were significantly lower in the malignancy group than in the non-malignancy group. In an additional analysis, pericardial fluid glucose levels were significantly lower in positive-cytology patients than in negative-cytology patients. However, neither pericardial fluid glucose levels nor the pericardial fluid-to-serum glucose ratio in our study was helpful for differentiating the malignancy group from the non-malignancy group because the areas under the receiver operating characteristic $(\mathrm{ROC})$ curve were $<0.7$. Pericardial fluid glucose level may decrease in diseases such as tuberculosis, rheumatism, and inflammatory diseases in non-malignancy patients, and serum glucose level can change secondary to various factors. In our study, no significant differences in the rates of diabetes mellitus and hypoglycemic agents were observed between the groups regarding pericardial fluid glucose levels.

CT attenuation values may be useful in determining the etiology of PE. We measured CT attenuation values in PE areas lager than the area in the ROI and used the former as the CT attenuation value. Previous studies reported that CT attenuation values were positively correlated with pleural and pericardial total protein levels and the pleural and pericardial/serum protein ratio [10, 19]. Moreover, Rifkin et al. demonstrated that CT attenuation values were strongly correlated with pericardial fluid hematocrit [20]. Our study showed that CT attenuation values were strongly correlated with pericardial fluid hematocrit, and that these values also had a good correlation with pericardial fluid glucose and protein levels, and glucose ratio and protein ratio. CT attenuation values from $20 \mathrm{HU}$ to $60 \mathrm{HU}$ indicated exudate in hypothyroidism, purulent pericarditis, or malignancy [21]. The median CT attenuation values in both groups was $>20 \mathrm{HU}$ for exudates, while the cutoff value was determined to be $20 \mathrm{HU}$ according to a previous report indicating that patients with CT attenuation values $>20 \mathrm{HU}$ had proteinaceous or hemorrhagic PE [22]. As a result, CT attenuation values $>20 \mathrm{HU}$ were associated with malignancy. Accordingly, the combination of pericardial fluid glucose $\leq 70 \mathrm{mg} / \mathrm{dl}$ and CT attenuation values $>20 \mathrm{HU}$ might be associated with malignancy in PE. In addition, this study showed that this combination was associated with positive-cytology patients.

Study limitations

There are several limitations to our study. First, the size of the non-malignancy group was small because this was a single-institution study. Second, the malignancy group had higher proportions of patients with lung cancer. 


\section{Conclusions}

Lower pericardial fluid glucose level in addition to higher CT attenuation values may suggest malignancyrelated PE. In patients with pericardial fluid glucose $\leq 70 \mathrm{mg} / \mathrm{dl}$ and CT attenuation values $>20 \mathrm{HU}$, malignant disease as the cause of PE must be excluded by other examinations and imaging modalities, even if there are no malignant cells in pericardial fluid cytology.

\section{Abbreviations}

CT: computed tomography; PE: pericardial effusion; LDH: lactate dehydrogenase; BNP: brain natriuretic peptide; HU: Hounsfield units; IQR: interquartile range; ADA: adenosine deaminase; UNL: upper limit of normal; ROI: region of interest: 2D: two-dimensional; CRP: C-reaction protein; Hb: hemoglobin; eGFR: estimated glomerular filtration rate; PET-CT: positron-emission tomography-CT; ROC: receiver operating characteristic

\section{Declarations}

\section{Ethics approval and consent to participate}

The study protocol was approved by the Ethics Committee of Kindai University Hospital. This study conformed to the ethical guidelines of the 1975 Declaration of Helsinki as reflected in a priori approval by our institution's human research committee. This was a retrospective observational study, with patient enrolment performed by the opt-out method on our hospital website (https://www.med.kindai.ac.jp/junnai/pdf/2020917\%20Nakamura.pdf). The informed consent waiver was approved by the Ethics Committee of Kindai University Hospital.

\section{Consent for publication}

Not applicable.

\section{Availability of data and materials}

The datasets used and/or analyzed during the current study are available from the corresponding author on reasonable request.

\section{Competing interests}

The authors declare that they have no competing interests.

\section{Funding}

None.

\section{Authors' contributions}


Takashi Nakamura: research ideas or designs, data collection, writing a treatise

Mana Okune: data collection, approval of final draft

Masakazu Yasuda, Heitaro Watanabe, Masafumi Ueno, Kenji Yamaji, Kazuki Mizutani: data analysis and interpretation, approval of final draft

Takashi Kurita: approval of final draft

Gaku Nakazawa: research ideas or designs, significant revision of the treatise, approval of final draft

\section{Acknowledgment}

We thank Jane Charbonneau, DVM, from Edanz Group (https://en-author-services.edanz.com/ac) for editing a draft of this manuscript.

\section{References}

1. Adler Y, Charron P, Imazio M, Badano L, Barón-Esquivias G, Bogaert J, et al; ESC Scientific Document Group. 2015 ESC Guidelines for the diagnosis and management of pericardial diseases: The Task Force for the Diagnosis and Management of Pericardial Diseases of the European Society of Cardiology (ESC) endorsed by: The European Association for Cardio-Thoracic Surgery (EACTS). Eur Heart J. 2015;36:2921-64.

2. Imazio $M$, Spodick $D H$, Brucato $A$, Trinchero R, Adler Y. Controversial issues in the management of pericardial diseases. Circulation. 2010;121:916-28.

3. Imazio M, Cecchi E, Demichelis B, lerna S, Demarie D, Ghisio A, et al. Indicators of poor prognosis of acute pericarditis. Circulation. 2007;115:2739-44.

4. El Haddad D, Iliescu C, Yusuf SW, William WN Jr, Khair TH, Song J, et al. Outcomes of cancer patients undergoing percutaneous pericardiocentesis for pericardial effusion. J Am Coll Cardiol. 2015;66:1119-28.

5. Gornik HL, Gerhard-Herman M, Beckman JA. Abnormal cytology predicts poor prognosis in cancer patients with pericardial effusion. J Clin Oncol. 2005;23:5211-6.

6. Albugami S, Al-Husayni F, AlMalki A, Dumyati M, Zakri Y, AlRahimi J. Etiology of pericardial effusion and outcomes post pericardiocentesis in the western region of Saudi Arabia: a single-center experience. Cureus. 2020;12:e6627.

7. Burazor I, Imazio M, Markel G, Adler Y. Malignant pericardial effusion. Cardiology. 2013;124:224-32.

8. Meyers DG, Meyers RE, Prendergast TW. The usefulness of diagnostic tests on pericardial fluid. Chest. 1997;111:1213-21.

9. Sogawa N, Michiue T, Kawamoto O, Oritani S, Ishikawa T, Maeda H. Postmortem virtual volumetry of the heart and lung in situ using CT data for investigating terminal cardiopulmonary pathophysiology in forensic autopsy. Leg Med (Tokyo). 2014;16:187-92. 
10. Çetin MS, Özcan Çetin EH, Özdemir M, Topaloğlu S, Aras D, Temizhan A, et al. Effectiveness of computed tomography attenuation values in characterization of pericardial effusion. Anatol $\mathrm{J}$ Cardiol. 2017;17:322-7.

11. Strobbe A, Adriaenssens T, Bennett J, Dubois C, Desmet W, McCutcheon K, et al. Etiology and longterm outcome of patients undergoing pericardiocentesis. J Am Heart Assoc. 2017;6:e007598.

12. Sullivan KM, Shulman HM, Storb R, Weiden PL, Witherspoon RP, McDonald GB, et. al. Chronic graftversus-host disease in 52 patients: adverse natural course and successful treatment with combination immunosuppression. Blood. 1981;57:267-76.

13. Mayosi BM. Contemporary trends in the epidemiology and management of cardiomyopathy and pericarditis in sub-Saharan Africa. Heart. 2007;93:1176-83.

14. Imazio M, Spodick DH, Brucato A, Trinchero R, Adler Y. Controversial issues in the management of pericardial diseases. Circulation. 2010;121:916-28.

15. LeWinter MM. Clinical practice. Acute pericarditis. N Engl J Med. 2014;371:2410-6.

16. Cheong XP, Law LKP, Seow SC, Tay LWE, Tan HC, Yeo WT, et al. Causes and prognosis of symptomatic pericardial effusions treated by pericardiocentesis in an Asian academic medical centre. Singapore Med J. 2020;61:137-41.

17. Ben-Horin S, Shinfeld A, Kachel E, Chetrit A, Livneh A. The composition of normal pericardial fluid and its implications for diagnosing pericardial effusions. Am J Med. 2005;118:636-40.

18. Karatolios K, Pankuweit S, Maisch B. Diagnostic value of biochemical biomarkers in malignant and non-malignant pericardial effusion. Heart Fail Rev. 2013;18:337-44.

19. Nandalur KR, Hardie AH, Bollampally SR, Parmar JP, Hagspiel KD. Accuracy of computed tomography attenuation values in the characterization of pleural fluid: an ROC study. Acad Radiol. 2005;12:987-91.

20. Rifkin RD, Mernoff DB. Noninvasive evaluation of pericardial effusion composition by computed tomography. Am Heart J. 2005;149:1120-7.

21. O'Leary SM, Williams PL, Williams MP, Edwards AJ, Roobottom CA, Morgan-Hughes GJ, et al. Imaging the pericardium: appearances on ECG-gated 64-detector row cardiac computed tomography. Br J Radiol. 2010;83:194-205.

22. Verhaert D, Gabriel RS, Johnston D, Lytle BW, Desai MY, Klein AL. The role of multimodality imaging in the management of pericardial disease. Circ Cardiovasc Imaging. 2010;3:333-43.

\section{Figures}


Figure 1


\section{Figure 1}

Measuring CT attenuation values in pericardial effusion. Two-dimensional images in three axial views: (a) upper slice: bifurcation of the pulmonary trunk, (b) middle slice: four-chamber heart view, (c) lower slice: upper edge of the liver. Both computed tomography (CT) attenuation values (in Hounsfield units $(\mathrm{HU})$ ) and pericardial effusion area were measured by plotting around the pericardial effusion in the three axial slices. The respective results were (a) 5022 mm2, 19.7 HU; (b) 6728 mm2, 18.4 HU; and (c) 12335 $\mathrm{mm} 2,17.7 \mathrm{HU}$. 
Figure 2


\section{Figure 2}

Comparison of the Pf glucose level and Pf/Se glucose ratio between the groups. Pericardial fluid glucose levels and pericardial fluid/serum glucose ratio were significantly lower in the malignancy group than in the non-malignancy group. Pf pericardial fluid, Se serum. 
Figure 3
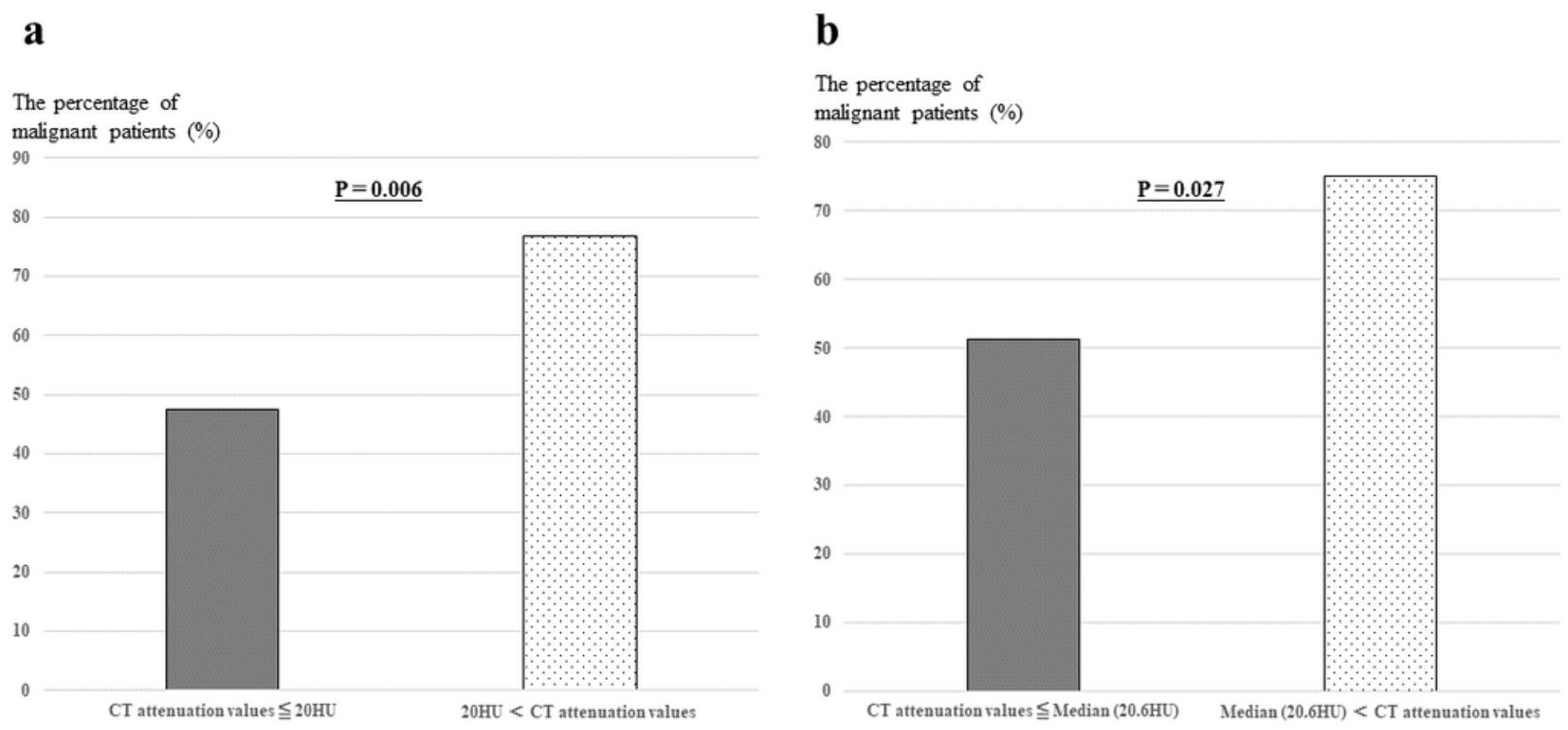

\section{Figure 3}

Correlation with CT attenuation values and the percentage of malignant patients. (a) Comparison between the percentage of malignant patients with computed tomography attenuation values $\leq 20$ Hounsfield units $(\mathrm{HU})$ vs the number of malignant patients with computed tomography (CT) attenuation values $<20 \mathrm{HU}$. (b) Comparison between the percentage of malignant patients with $\mathrm{CT}$ attenuation values $\leq$ the median and the number of malignant patients with values $<$ the median $\mathrm{CT}$ attenuation value. 


\section{Figure 4}
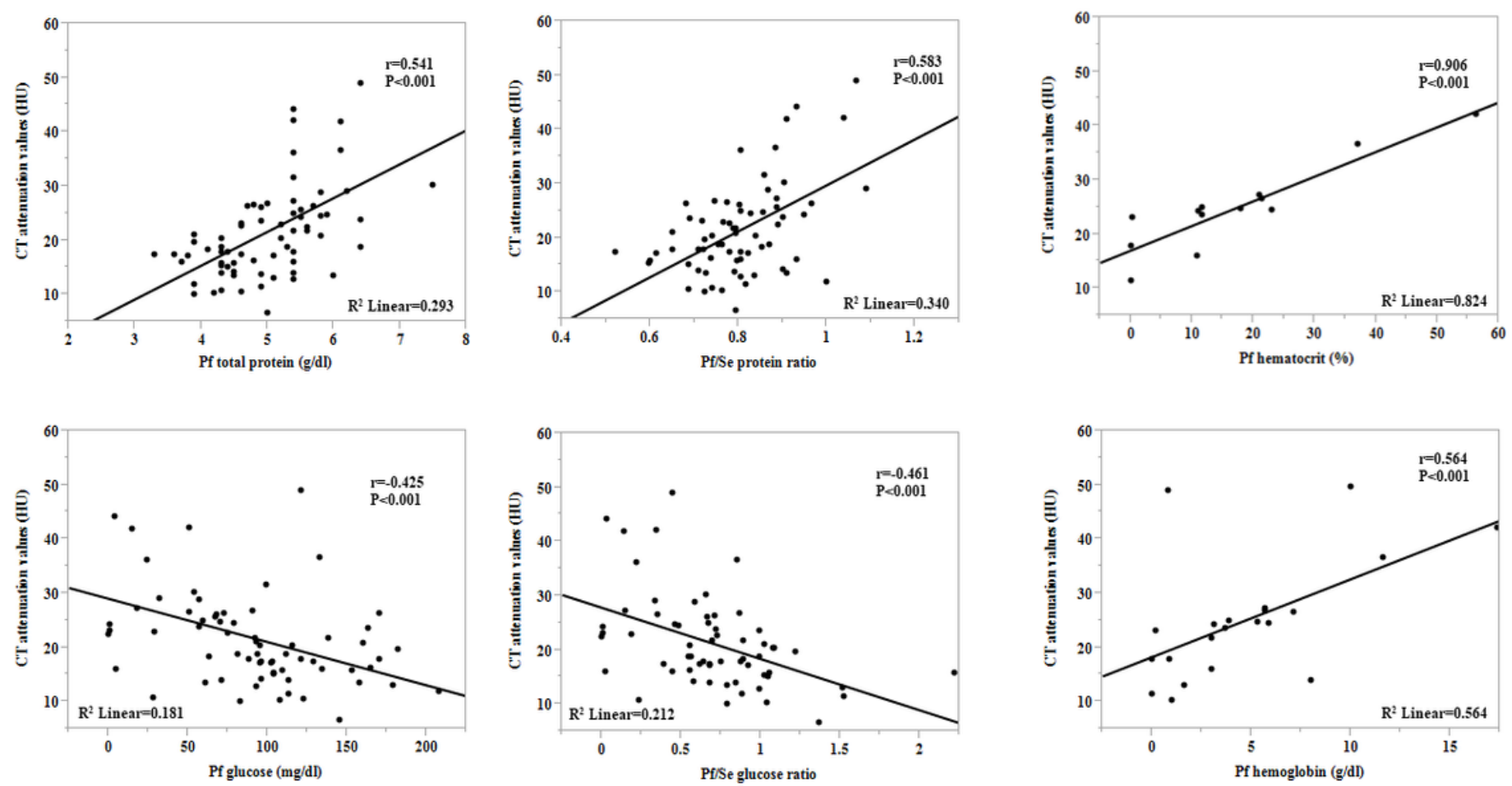

\section{Figure 4}

Correlation between CT attenuation values and pericardial fluid parameters. The pericardial fluid parameters were: total protein level, protein ratio, glucose level, glucose ratio, hematocrit, and hemoglobin. Each dot represents one patient; the straight line represents the best fit line obtained by linear regression analysis. Pf pericardial fluid, Se serum, HU Hounsfield units, CT computed tomography. 
Figure 5

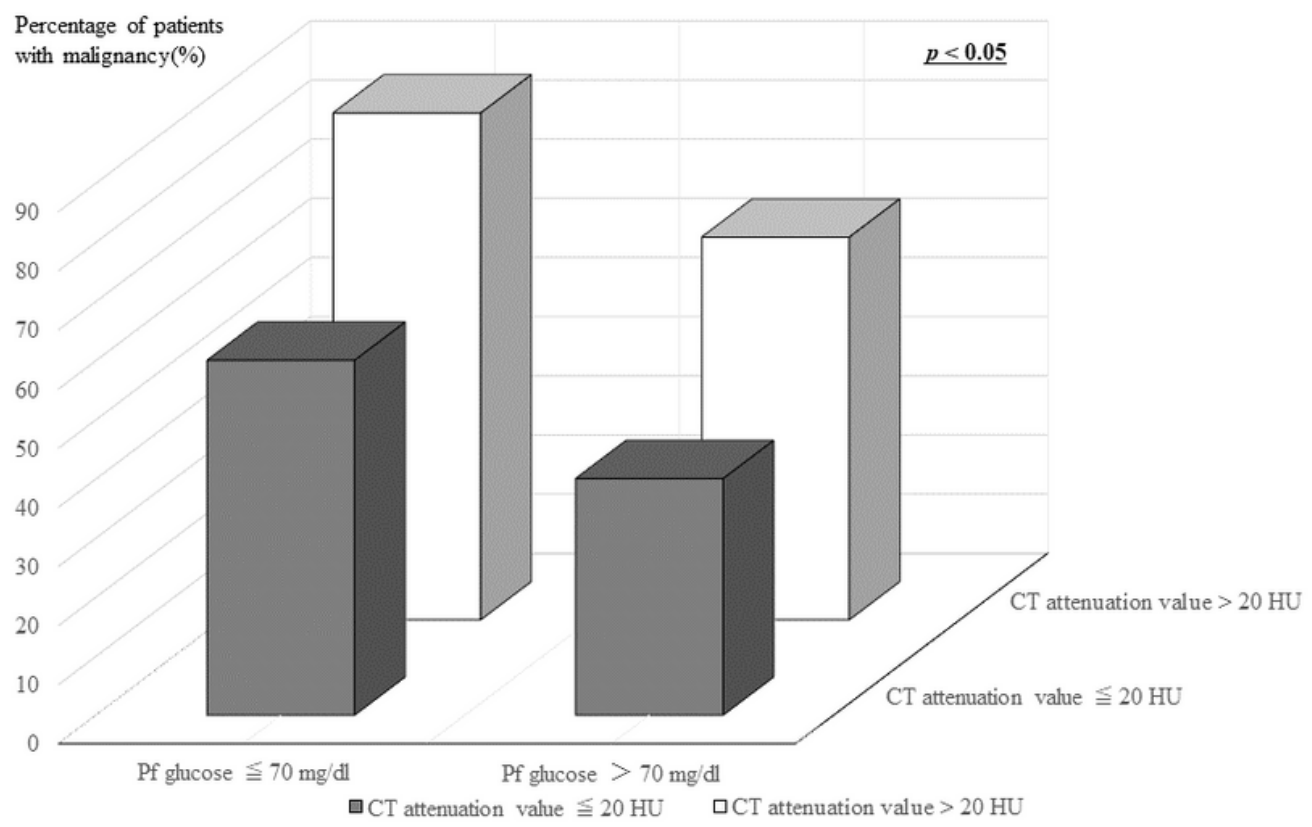

\section{Figure 5}

Relationship between pericardial fluid glucose or CT attenuation values and malignancy vs nonmalignancy. Pericardial fluid glucose $\leq 70 \mathrm{mg} / \mathrm{dl}$ and CT attenuation values $>20 \mathrm{HU}$ were cutoff values associated with malignancy. Pf pericardial fluid, CT computed tomography.

\section{Supplementary Files}

This is a list of supplementary files associated with this preprint. Click to download.

- AdditionalFile1.pptx 